\title{
Derivation of amplitude equations for nonlinear oscillators subject to arbitrary forcing
}

\author{
Catalina Mayol, Raúl Toral, and Claudio R. Mirasso \\ Department de Física, Universitat de les Illes Balears, and Instituto Mediterráneo de Estudios Avanzados (IMEDEA), CSIC-UIB, \\ Ed. Mateu Orfila, Campus UIB, 07122 Palma de Mallorca, Spain*
}

(Received 4 December 2003; published 24 June 2004)

\begin{abstract}
By using a generalization of the multiple scales technique we develop a method to derive amplitude equations for zero-dimensional forced systems. The method allows to consider either additive or multiplicative forcing terms and can be straightforwardly applied to the case that the forcing is white noise. We give examples of the use of this method to the case of the van der Pol-Duffing oscillator. The writing of the amplitude equations in terms of a Lyapunov potential allow us to obtain an analytical expression for the probability distribution function which reproduces reasonably well the numerical simulation results.
\end{abstract}

DOI: 10.1103/PhysRevE.69.066141

PACS number(s): 05.10.Gg, 02.30.Mv, 05.40.Ca

\section{INTRODUCTION}

It is known that the general trends of the behavior of a dynamical system can be captured, in some cases of interest, by the so-called amplitude equations [1,2], describing the slow dynamics of the envelope of the trajectories. One of the most interesting features of amplitude equations is their universality: many systems can share the same amplitude equation depending only on general symmetry considerations. Another advantage of using the amplitude equations is that they allow numerical integration with a bigger integration step. A very successful technique, amongst others, for the derivation of amplitude equations is that of the multiple scales method. This technique has been extensively applied, for instance, to both unforced and periodically forced nonlinear oscillators $[1,3,4]$. However, for more general, e.g., nonperiodic, forcing terms there is no systematic derivation of the amplitude equation. In this paper we develop a possible extension of the multiple scales method to obtain amplitude equations for dynamical systems forced with general functions. As an application, we consider oscillators which are randomly forced either by additive noise [5-9] or by multiplicative noise $[6,10-12]$.

The derivation of amplitude equations for randomly forced dynamical systems has been previously considered in the literature. In some works, dynamical systems are treated in a probabilistic way and noise effects on bifurcations are studied [13-16]. A different approach has been used in Refs. $[17,18]$ where the authors derive a stochastic Landau form as the amplitude equation for the stochastic Swift-Hohenberg model and use it to describe the dynamics of the bifurcating solutions. A simplifying feature of this case is that the SwiftHohenberg equation has only first-order derivatives in time. Second order equations have been studied in Ref. [19] at the level of probability distribution functions. Our method, being a straightforward extension of the multiple scales method, can be easily applied to dynamical systems including second-order time derivatives.

This paper is structured as follows. In Sec. II, the general theory is presented using the van der Pol-Duffing oscillator

*URL: http://www.imedea.uib.es with an arbitrary forcing term as an example. In Sec. III, the same oscillator is considered under the influence of an additive noise forcing term. In Sec. IV, we present the results for the multiplicative noise case. Finally, the main conclusions of this paper are reviewed in Sec. V.

\section{GENERAL THEORY}

Although we believe our method to be quite general, for the sake of concreteness, in this section we study an oscillatory system, namely, a van der Pol-Duffing oscillator, with a general additive forcing term, as defined by the following dimensionless equation for the variable $x(t)$ :

$$
\ddot{x}+x=\epsilon\left[k_{1}\left(1-x^{2}\right) \dot{x}-k_{2} x^{3}\right]+\epsilon f(t),
$$

being $f(t)$ any time dependent function and $\epsilon$ is considered as a small parameter. The specific cases $k_{1}=1, k_{2}=0$ (van der Pol oscillator) and $k_{1}=0, k_{2}=1$ (Duffing oscillator) are contained in this general equation. For $f(t)=0$, the van der PolDuffing oscillator has an unstable fixed point and a stable limit cycle in the phase space $(x, \dot{x})$. Equation parameters have been rescaled out such that the frequency of the linear oscillator, i.e., $\epsilon=0$, is $\omega_{0}=1$. For $\epsilon>0$, the evolution can be written as $x(t)=A(t) e^{i \omega_{0} t}+\bar{A}(t) e^{-i \omega_{0} t}$, being $A(t)$ the slowly varying complex amplitude $(\bar{A}$ denotes the complex conjugate of $A$ ).

We extend the method of multiple scales in order to be able to consider Eq. (1) for a general function $f(t)$. Let us briefly review the method of multiple scales [1]. In this method, one looks for a series expansion of the time dependent variable $x(t)$ of the form

$$
x(t)=x_{0}(t)+\epsilon x_{1}(t)+\epsilon^{2} x_{2}(t)+\cdots .
$$

The main point is to consider different time scales: $T_{0}, T_{1}, T_{2}, \cdots$, with $T_{m}=\epsilon^{m} t$ as independent variables and hence any function of time becomes a function of the $T_{m}$ 's:

$$
\begin{aligned}
& x_{0}(t)=x_{0}\left(T_{0}, T_{1}, \ldots\right), \\
& x_{1}(t)=x_{1}\left(T_{0}, T_{1}, \ldots\right),
\end{aligned}
$$




$$
\begin{gathered}
x_{2}(t)=x_{2}\left(T_{0}, T_{1}, \ldots\right) \\
\vdots \\
f(t)=\hat{f}\left(T_{0}, T_{1}, \ldots\right) .
\end{gathered}
$$

The notation $\hat{f}\left(T_{0}, T_{1}, \ldots\right)$ stresses the fact that, although equal in value, both functions $f$ and $\hat{f}$ are formally different since they contain a different number of variables. Note that the decomposition of $f(t)$ into $\hat{f}\left(T_{0}, T_{1}, \ldots\right)$ is not unique, as we will see in the following examples. The time derivative is transformed according to

$$
\frac{d}{d t}=\frac{\partial}{\partial T_{0}}+\epsilon \frac{\partial}{\partial T_{1}}+\epsilon^{2} \frac{\partial}{\partial T_{2}}+\cdots=D_{0}+\epsilon D_{1}+\epsilon^{2} D_{2}+\cdots
$$

where $D_{i} \equiv \partial / \partial T_{i}$. By substituting this expansion into Eq. (1) and equating coefficients up to order $\epsilon^{1}$, one obtains

$$
\left(D_{0}^{2}+1\right) x_{0}=0
$$

$$
\left(D_{0}^{2}+1\right) x_{1}=k_{1}\left(1-x_{0}^{2}\right) D_{0} x_{0}-k_{2} x_{0}^{3}-2 D_{0} D_{1} x_{0}+\hat{f}\left(T_{0}, T_{1}, \ldots\right) .
$$

The solution of Eq. (8) is

$$
x_{0}\left(T_{0}, T_{1}, \ldots\right)=A\left(T_{1}\right) e^{i T_{0}}+\bar{A}\left(T_{1}\right) e^{-i T_{0}},
$$

where it has been assumed that the amplitude $A\left(T_{1}\right)$ of the sinusoidal solution depends only on the slow time variable $T_{1}$ instead of all the sequence $T_{1}, T_{2}, \ldots$. Accordingly, at this order, $f(t)=\hat{f}\left(T_{0}, T_{1}\right)$. Replacing this solution in Eq. (9), we get

$$
\begin{aligned}
\left(D_{0}^{2}+1\right) x_{1}= & {\left[i k_{1} A\left(1-|A|^{2}\right)-2 i A^{\prime}-3 k_{2} A|A|^{2}\right] e^{i T_{0}} } \\
& +\left[-i k_{1} \bar{A}\left(1-|A|^{2}\right)+2 i \bar{A}^{\prime}-3 k_{2} A|A|^{2}\right] e^{-i T_{0}} \\
& -i A^{3} e^{3 i T_{0}}+i \bar{A}^{3} e^{-3 i T_{0}}+\hat{f}\left(T_{0}, T_{1}\right) \equiv g\left(T_{0}, T_{1}\right),
\end{aligned}
$$

where $A^{\prime}$ denotes the derivative of $A$ with respect to $T_{1}$, and we define $g\left(T_{0}, T_{1}\right)$ as the right-hand side of the equation. The amplitude equation is obtained by avoiding resonant terms with the frequency of the left-hand side of the equation,

$$
\left\langle e^{i T_{0}} \mid g\left(T_{0}, T_{1}\right)\right\rangle=0,
$$

with the scalar product for the $T_{0}$ variable defined as

$$
\left\langle v\left(T_{0}\right) \mid w\left(T_{0}\right)\right\rangle=\int_{-\infty}^{\infty} \bar{v}\left(T_{0}\right) w\left(T_{0}\right) d T_{0},
$$

and the result $\left\langle e^{i n T_{0}} \mid e^{i m T_{0}}\right\rangle=\delta_{n, m}$. By using Eqs. (11) and (12) one obtains

$$
i\left[k_{1} A\left(1-|A|^{2}\right)-2 A^{\prime}\right]-3 k_{2} A|A|^{2}+\left\langle e^{i T_{0}} \mid \hat{f}\left(T_{0}, T_{1}\right)\right\rangle=0 .
$$

The problem has been reduced to extract from $f(t)$ $=\hat{f}\left(T_{0}, T_{1}\right)$ the resonant terms, i.e., those with a component of $e^{i T_{0}}$. These terms are those giving a contribution different from zero in the previous equation.

The particular case of a periodic forcing term, $f(t)$ $=\cos (\lambda t)$, with a forcing frequency $\lambda=\omega_{0}+\sigma \epsilon=1+\sigma \epsilon$, and $\sigma=O(1)$ (soft resonant excitation) has been extensively studied in the past. In this case, the standard approach [1] considers the decomposition,

$$
\begin{aligned}
\cos [(1+\sigma \epsilon) t)= & \cos (t+\sigma \epsilon t)=\cos \left(T_{0}+\sigma T_{1}\right)=\frac{1}{2}\left(e^{i T_{0}} e^{i \sigma T_{1}}\right. \\
& \left.+e^{-i T_{0}} e^{-i \sigma T_{1}}\right)
\end{aligned}
$$

When substituting this expression in Eq. (14) for the $A$ variable, the term multiplying $e^{i T_{0}}$ is the only one giving a nonzero contribution. In other words, the contribution to the equation for $A$ is the spectral component of the function $f(t)$ at the frequency $\omega_{0}=1$. The resulting amplitude equation is

$$
\frac{d A}{d T_{1}}=k_{1} \frac{1}{2} A\left(1-|A|^{2}\right)+i k_{2} \frac{3}{2} A|A|^{2}-\frac{i}{4} e^{i \sigma T_{1}},
$$

where it appears that the proper time scale for the variation of the amplitude $A$ is given by $T_{1}$.

As it is clear in this and other examples, the derivation of the amplitude equation requires the evaluation of expressions of the general form,

$$
\left\langle e^{i T_{0}} \mid e^{i m T_{0}} \hat{f}\left(T_{0}, T_{1}\right)\right\rangle,
$$

for which Eq. (14) is a particular case with $m=0$. As stated before, the problem reduces then to find a suitable expression for $\hat{f}\left(T_{0}, T_{1}\right)$. Some particular functions might allow a "natural" decomposition, e.g., Eq. (15). However, for an arbitrary $f(t)$ the splitting in terms of slow, $T_{0}$, and fast, $T_{1}$, variables might not be so straightforward. Our proposal is to make the following decomposition:

$$
\hat{f}\left(T_{0}, T_{1}\right)=e^{-(m-1) i T_{0}} e^{i(m-1) T_{1} / \epsilon} f\left(T_{1} / \epsilon\right),
$$

which allows to compute Eq. (17) as

$$
\left\langle e^{i T_{0}} \mid e^{i m T_{0}} \hat{f}\left(T_{0}, T_{1}\right)\right\rangle=e^{i(m-1) T_{1} / \epsilon} f\left(T_{1} / \epsilon\right) .
$$

This simple rule has to be applied with the necessary values of $m$ as demanded in each case. Notice that this decomposition gives always a nonvanishing contribution to the scalar product. This is particularly interesting in the case of nearly constant functions $f(t)$ for which the spectrum has a peak at $\omega=0$, far from the main peaks at $\pm \omega_{0}$. In the white noise case, our proposal is able to extract a suitable contribution from the flat spectrum.

Coming back to our example, Eq. (14) contains a term of the form (17) with $m=0$. Substitution of the ansatz Eq. (19) yields 
a)

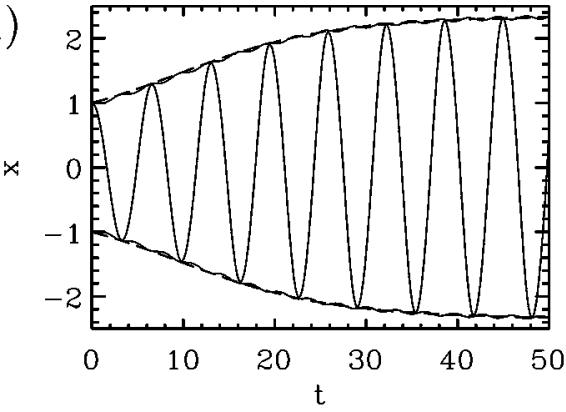

b)

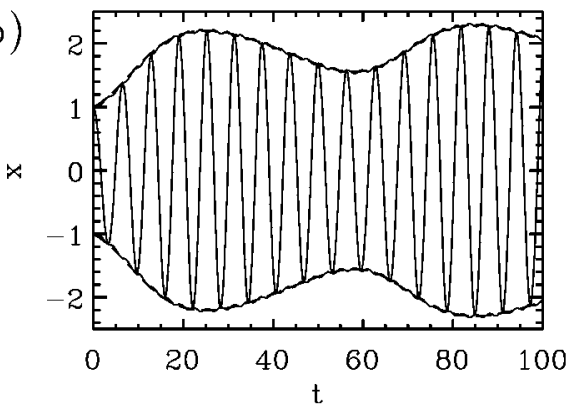

c)

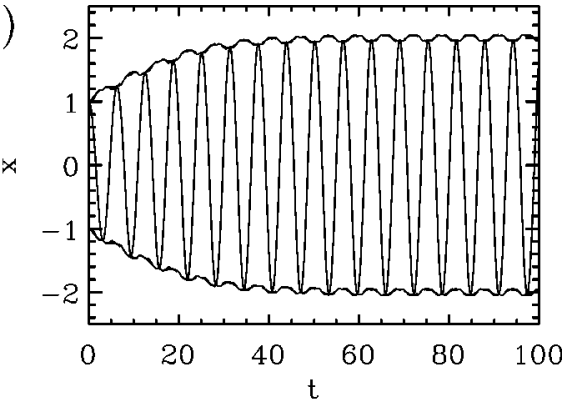

d)

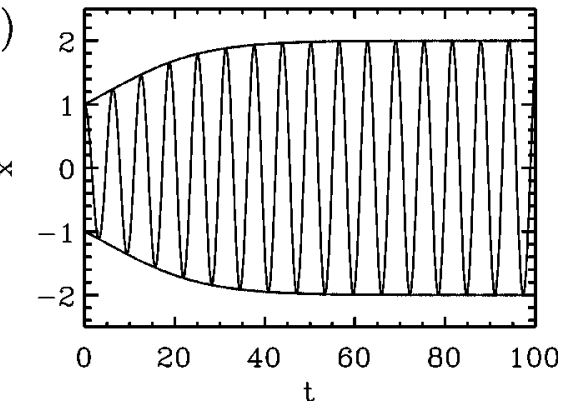

FIG. 1. Time evolution of $x(t)$ in a van der Pol oscillator (1) (oscillating thin solid line) forced with $f(t)=\cos [(1+\sigma \epsilon) t]$. In order to extract the envelope from the complex amplitude $A$ we also plot $\pm 2 R$, where $A=R e^{i \phi}$ in two cases: the solid line is the result of method (21), the dashed line comes from the standard method (16). Notice that both methods give almost indistinguishable results. The values of the parameters are $\epsilon=0.1, k_{1}=1, k_{2}=0$. The initial conditions: $x(0)=1, \dot{x}(0)=0$ are used henceforth in all the examples given. The different plots are: (a) $\sigma=0$; (b) $\sigma=1$; (c) $\sigma=10$; (d) $\sigma=500$.

$$
\frac{d A}{d T_{1}}=k_{1} \frac{A}{2}\left(1-|A|^{2}\right)+i k_{2} \frac{3}{2} A|A|^{2}-\frac{i}{2} e^{-i T_{1} / \epsilon} f\left(T_{1} / \epsilon\right) .
$$

This is our result for the amplitude equation of the van der Pol-Duffing oscillator under general forcing $f(t)$. Concretely, the choice $f(t)=\cos [(1+\sigma \epsilon) t]$ gives

$$
\frac{d A}{d T_{1}}=k_{1} \frac{A}{2}\left(1-|A|^{2}\right)+i k_{2} \frac{3}{2} A|A|^{2}-\frac{i}{4} e^{i \sigma T_{1}}-\frac{i}{4} e^{-i \sigma T_{1}} e^{-i 2 T_{1} / \epsilon}
$$

As compared to the standard result of Eq. (16), this equation includes an extra term that gives oscillations in the amplitude at the scale $T_{1} / \epsilon$. However, in this case these oscillations belong to the fast time scale $T_{0}$ and could, in principle, be eliminated thus leading to the standard result, Eq. (16). Indeed, as shown in Fig. 1, the effect of this extra term is very small and decreases with increasing $\sigma$.

We now consider the forcing term $f(t)=f_{0}$, constant, for which the standard method does not obtain any contribution. Our method immediately yields

$$
\frac{d A}{d T_{1}}=k_{1} \frac{A}{2}\left(1-|A|^{2}\right)+i k_{2} \frac{3}{2} A|A|^{2}-\frac{i}{2} e^{-i T_{1} / \epsilon} f_{0} .
$$

One could again decide to eliminate the oscillations in the fast scale $T_{1} / \epsilon$. However, as shown in Fig. 2, the influence of the extra term can actually improve upon the predictions of the amplitude equation. In particular, it can describe the lack of symmetry around the zero value observed in the evolution of $x(t)$.
The above scheme can be applied to the case of multiplicative forcing term. Again, we consider a specific example; the van der Pol-Duffing equation with a linear multiplicative term of the form

$$
\ddot{x}+x=\epsilon\left[k_{1}\left(1-x^{2}\right) \dot{x}-k_{2} x^{3}\right]+\epsilon x f(t) .
$$

The multiple scales ansatz (2) leads in the first-order approximation to the form (10). The next order yields

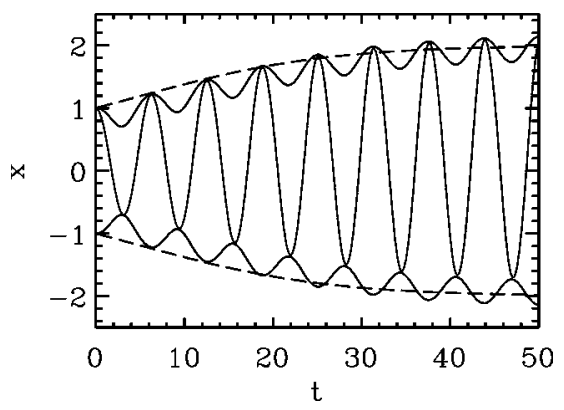

FIG. 2. Time evolution of $x(t)$ in a van der Pol oscillator (1) (oscillating thin solid line) forced with $f(t)=2.0$. We also plot the envelope $\pm 2 R(t)$ as coming from Eq. (22) (solid line). The parameters are $\epsilon=0.1, k_{1}=1, k_{2}=0$. Notice that the fit of the envelope to the trajectory of $x(t)$ worsens if we neglect the last term in Eq. (22), as shown by the dashed line, specially in the negative values of $x(t)$. 


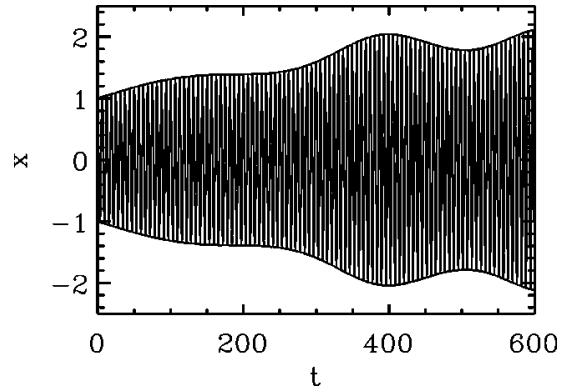

FIG. 3. Time evolution of $x(t)$ in a van der Pol-Duffing oscillator (23) (oscillating thin solid line) with multiplicative forcing $x f(t)=x \cos (2 t)$, together with the envelope $\pm 2 R(t)$ coming both from Eqs. (26) and (27) (they are indistinguishable at the scale of the figure). The values of the parameters are $k_{1}=1, k_{2}=1, \epsilon=0.01$.

$$
\begin{aligned}
D_{0}^{2} x_{1}+x_{1}= & k_{1}\left(1-x_{0}^{2}\right) D_{0} x_{0}-k_{2} x_{0}^{3}-2 D_{0} D_{1} x_{0}+x_{0} \hat{f}\left(T_{0}, T_{1}\right) \\
= & e^{i T_{0}}\left[-2 i \frac{\partial A}{\partial T_{1}}+i k_{1} A-i k_{1} A|A|^{2}-3 k_{2}|A|^{2} A\right. \\
& \left.+\hat{f}\left(T_{0}, T_{1}\right) A\right]+e^{-i T_{0}}\left[2 i \frac{\partial \bar{A}}{\partial T_{1}}-i k_{1} \bar{A}+i k_{1} \bar{A}|A|^{2}\right. \\
& \left.-3 k_{2}|A|^{2} \bar{A}+\hat{f}\left(T_{0}, T_{1}\right) \bar{A}\right]-e^{3 i T_{0}} A^{3}\left(i k_{1}+k_{2}\right) \\
& +e^{-3 i T_{0}} \bar{A}^{3}\left(i k_{1}-k_{2}\right) \equiv g_{1}\left(T_{0}, T_{1}\right)
\end{aligned}
$$

The nullity of the scalar product $\left\langle e^{i T_{0}} \mid g_{1}\left(T_{0}, T_{1}\right)\right\rangle=0$ involves two contributions of the form (17): one with $m=1$ and another with $m=-1$. These contributions are computed according to the general rule (19). The amplitude equation obtained is

$$
\begin{aligned}
\frac{d A}{d T_{1}}= & k_{1} \frac{1}{2} A\left(1-|A|^{2}\right)+i k_{2} \frac{3}{2} A|A|^{2}-\frac{i}{2} A f\left(T_{1} / \epsilon\right) \\
& -\frac{i}{2} \bar{A} e^{-2 i T_{1} / \epsilon} f\left(T_{1} / \epsilon\right) .
\end{aligned}
$$

Let us be more specific and consider $f(t)=\cos (2 t)$. The amplitude equation is

$$
\begin{aligned}
\frac{d A}{d T_{1}=} & k_{1} \frac{1}{2} A\left(1-|A|^{2}\right)+i k_{2} \frac{3}{2} A|A|^{2}-\frac{i}{4} \bar{A}-\frac{1}{2} \bar{A} \cos \left(\frac{2 T_{1}}{\epsilon}\right) \\
& -\frac{i}{4} \bar{A} e^{-4 i T_{1} / \epsilon} .
\end{aligned}
$$

Again the last two terms, belonging to the temporal scale $T_{0}$, could be discarded reobtaining the amplitude equation derived after replacing directly $\cos (2 t)=\cos \left(2 T_{0}\right)$ in Eq. (24),

$$
\frac{d A}{d T_{1}}=k_{1} \frac{1}{2} A\left(1-|A|^{2}\right)+i k_{2} \frac{3}{2} A|A|^{2}-\frac{i}{4} \bar{A} .
$$

In Fig. 3 we compare the results of Eqs. (23), (26), and (27). Observe that both amplitude equations faithfully follow the envelope of the $x(t)$ variable.

\section{ADDITIVE NOISE}

We apply the above developed technique to the case of an additive noise forcing term, while in a following section we will consider multiplicative noise. We take equation (1) with a real noise term $f(t)=\xi(t)$ with zero mean value and temporal correlations $\left\langle\xi(t) \xi\left(t^{\prime}\right)\right\rangle=\delta\left(t-t^{\prime}\right)$. The general amplitude equation (20) adopts now the form

$$
\frac{d A}{d T_{1}}=k_{1} \frac{A}{2}\left(1-|A|^{2}\right)+i k_{2} \frac{3}{2} A|A|^{2}-\frac{i}{2} e^{-i T_{1} / \epsilon} \sqrt{\epsilon} \xi\left(T_{1}\right),
$$

with $\left\langle\xi\left(T_{1}\right) \xi\left(T_{1}^{\prime}\right)\right\rangle=\delta\left(T_{1}-T_{1}^{\prime}\right)$, and it has been used that $\xi\left(T_{1} / \epsilon\right)=\sqrt{\epsilon} \xi\left(T_{1}\right)$. The statistical properties that follow from this equation are contained in the corresponding FokkerPlanck equation for the time evolution of the probability density function $P(A, t)[20]$. It is possible to obtain an approximate analytical expression in the steady state $P_{s t}(A)$. We first write down the real and imaginary parts of the amplitude equation,

$$
\begin{aligned}
\frac{d A_{r}}{d T_{1}}= & \frac{k_{1}}{2} A_{r}\left[1-\left(A_{r}^{2}+A_{i}^{2}\right)\right]-k_{2} \frac{3}{2} A_{i}\left(A_{r}^{2}+A_{i}^{2}\right) \\
& -\frac{1}{2} \sin \left(T_{1} / \epsilon\right) \sqrt{\epsilon} \xi\left(T_{1}\right), \\
\frac{d A_{i}}{d T_{1}}= & \frac{k_{1}}{2} A_{i}\left[1-\left(A_{r}^{2}+A_{i}^{2}\right)\right]+k_{2} \frac{3}{2} A_{r}\left(A_{r}^{2}+A_{i}^{2}\right) \\
& -\frac{1}{2} \cos \left(T_{1} / \epsilon\right) \sqrt{\epsilon} \xi\left(T_{1}\right),
\end{aligned}
$$

with $A=A_{r}+i A_{i}$. It can be shown that the deterministic terms are that of a nonrelaxational potential flow with the Lyapunov function [21,22],

$$
V(A)=k_{1}\left[-\frac{|A|^{2}}{4}+\frac{|A|^{4}}{8}\right] .
$$

Using this fact, one can obtain an approximate analytical expression for the stationary probability distribution $P_{s t}(A)$. To this end, we simplify the stochastic set of Eqs. (29) by substituting the cosine and sine functions by its root-mean square value $\left\langle\sin ^{2}(t)\right\rangle^{1 / 2}=\left\langle\cos ^{2}(t)\right\rangle^{1 / 2}=1 / \sqrt{2}$ and considering that $\langle\sin (t) \cos (t)\rangle=0$, effectively introducing different noise terms for the real and imaginary parts. This yields

$$
\frac{d A}{d T_{1}}=\frac{k_{1}}{2} A\left[1-|A|^{2}\right]+i k_{2} \frac{3}{2} A|A|^{2}-\frac{1}{2} \sqrt{\frac{\epsilon}{2}} \eta\left(T_{1}\right),
$$

where $\eta$ is a complex noise term with zero mean value and correlations $\left\langle\eta\left(T_{1}\right) \bar{\eta}\left(T_{1}^{\prime}\right)\right\rangle=\delta\left(T_{1}-T_{1}^{\prime}\right)$. It turns out that this new noise terms satisfy the fluctuation-dissipation relation [21], and the stationary probability distribution is given in terms of the Lyapunov potential as

$$
P_{s t}(A) \propto \exp (-V(A) / \widetilde{\epsilon})
$$

where $\tilde{\epsilon}=\epsilon / 16$. 


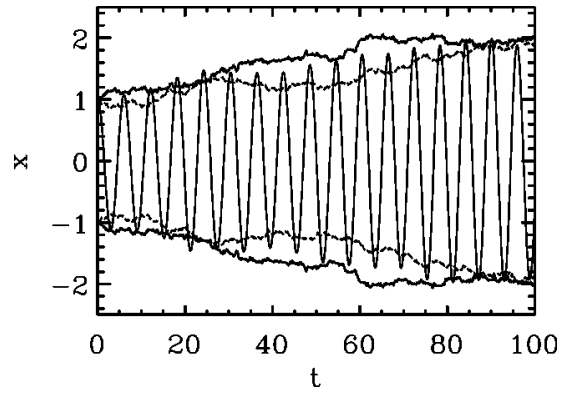

FIG. 4. Time evolution of $x(t)$ in a van der Pol-Duffing oscillator (1) (oscillating thin solid line) forced with additive white noise $f(t)=\xi(t)$, and the envelope $\pm 2 R(t)$ coming from Eq. (29) (solid line) and from the approximated expression (31) (dashed line). The parameters are $k_{1}=1, k_{2}=1, \epsilon=0.1$. Notice that the individual trajectories are not exactly approximated by the amplitude equations, while the mean values would be (see next figure).

We have simulated the dynamics of Eq. (1) with $f(t)$ $=\xi(t)$ following standard stochastic integration methods as given in Ref. [21]. The results are compared with the corresponding amplitude equation, i.e., Eq. (29), in Fig. 4. We observe that the amplitude equation does not fit the actual maxima $x_{\max }$ of $x(t)$ for a given realization. However, by taking many realizations, the averaged amplitude does fit the maxima of the average values. As an evidence, we show in Fig. 5, the histogram for the $x_{\max }$ is compared to the histogram for the amplitude. In solid line, it appears the approximate theoretical expression (32). The concordance between solid line and triangles reveals the validity of the approximation which allowed us to derive Eq. (31) from Eq. (29).

\section{MULTIPLICATIVE NOISE}

In this section we consider the van der Pol oscillator with a multiplicative noise term of the form $f(t)=x \xi(t)$. The corresponding amplitude equation (25) becomes

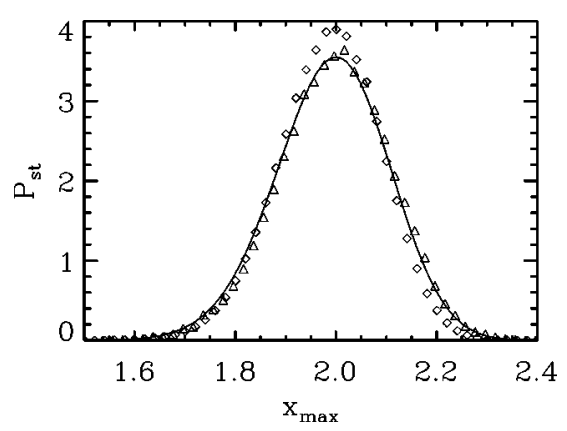

FIG. 5. The diamonds correspond to the histogram for the maxima of $x$ for a van der Pol-Duffing oscillator in the same case of additive white noise forcing and parameters than in the previous Fig. 4. The triangles are the histogram of the envelope $2 R(t)$ as obtained from the amplitude equation (29). The solid line is the probability distribution function as given by the approximate analytical result of Eq. (32).

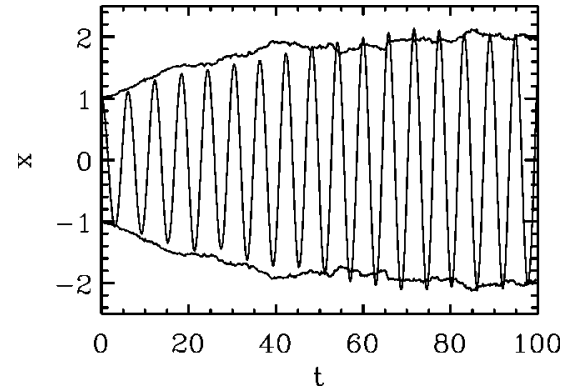

FIG. 6. Time evolution $x(t)$ for a van der Pol-Duffing oscillator (thin solid oscillating line) with a multiplicative noise term $x f(t)$ $=x \xi(t)$, and the envelope $\pm 2 R(t)$ coming from the amplitude equation (33) (solid line). The parameters are $k_{1}=1, k_{2}=1, \epsilon=0.1$.

$$
\begin{aligned}
\frac{d A}{d T_{1}}= & k_{1} \frac{1}{2} A\left(1-|A|^{2}\right)+i k_{2} \frac{3}{2} A|A|^{2}-\frac{i}{2} A \sqrt{\epsilon} \xi\left(T_{1}\right) \\
& -\frac{i}{2} \bar{A} e^{-2 i T_{1} / \epsilon} \sqrt{\epsilon} \xi\left(T_{1}\right) .
\end{aligned}
$$

In this case of multiplicative noise terms, the time traces are similar to the ones observed for additive noise terms, see Fig. 6.

In order to be able to compute the stationary probability distribution, we now introduce modulus and phase variables: $A=R e^{i \phi}$, the resulting equations are

$$
\begin{gathered}
\frac{d R}{d T_{1}}=k_{1} \frac{1}{2} R\left(1-R^{2}\right)-\frac{1}{2} R \xi\left(T_{1} / \epsilon\right) \sin \left(2\left[T_{1} / \epsilon+\phi\right]\right), \\
\frac{d \phi}{d T_{1}}=k_{2} \frac{3}{2} R^{2}-\frac{1}{2} \xi\left(T_{1} / \epsilon\right)-\frac{1}{2} \xi\left(T_{1} / \epsilon\right) \cos \left(2\left[T_{1} / \epsilon+\phi\right]\right) .
\end{gathered}
$$

Again, we approximate the sine and cosine terms by its rootmean-square value. In this way, the equation for the modulus does not contain the phase variable,

$$
\frac{d R}{d T_{1}}=k_{1} \frac{1}{2} R\left(1-R^{2}\right)-\frac{1}{2} R \sqrt{\frac{\epsilon}{2}} \xi\left(T_{1}\right) .
$$

This equation can be analyzed using the general methods $[21,23,24]$ and it can be shown that, despite the fact that the Lyapunov function does not satisfy the fluctuationdissipation relation, Eq. (32) still holds asymptotically in the limit $\epsilon \rightarrow 0$ giving

$$
P_{s t}(R) \propto \exp \left(-\frac{4 k_{1}}{\epsilon}\left[-R^{2}+\frac{R^{4}}{2}\right]\right) .
$$

This result is compared in Fig. 7 with the numerical simulations.

We would like to end this section by commenting that Eq. (35) has the same structure that Eq. (35) of Ref. [19], in the sense that the noise term $\xi\left(T_{1}\right)$ appears multiplying the variable $R$. In that reference, the result is obtained by working at the level of the probability distribution function. Our 


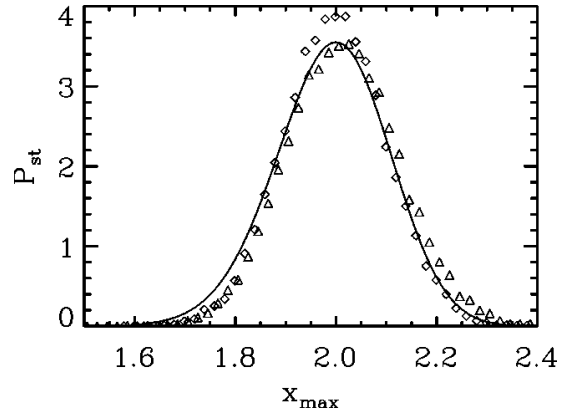

FIG. 7. The diamonds correspond to the histogram for the maxima of $x$ for a van der Pol-Duffing oscillator in the same case of multiplicative white noise forcing and parameters than in the previous Fig. 5. The triangles are the histogram of the envelope $2 R(t)$ as obtained with the amplitude equation (33). The solid line is the probability distribution function as given by the approximate analytical result of Eq. (36).

method, which we believe to be simpler and more straightforward, works directly at the level of the (stochastic) dynamical equation.

\section{CONCLUSIONS}

In this work, we develop a simple, general method to obtain amplitude equations for a large variety of dynamical systems forced by an arbitrary time dependent function $f(t)$ including noises, both additive and multiplicative. Our method is based on the multiple scales analysis combined with a recipe to extract the resonant terms for arbitrary forcing functions. As a representative example, we analyze in detail the van der Pol-Duffing oscillator.

In the deterministic case, our method is able to reproduce standard results. It only differs from them in the presence of some extra terms in the amplitude equations. We show that those terms are either very small or improve upon the predictions of the equation. For example, it can incorporate the asymmetries observed in the simulations, as in Fig. 2.

In the stochastic additive case, $f(t)=\xi(t)$, our method is able to satisfactorily capture its contribution to the amplitude equation. A simple set of approximations allows us to obtain analytically the stationary probability distribution $P_{s t}(A)$ in terms of a Lyapunov potential function.

Similar conclusions can be drawn for the multiplicative noise, $f(t)=x \xi(t)$. Again, our method allows us to obtain easily the stochastic amplitude equation. An approximation, valid now in the limit of small noise, yields the stationary distribution.

\section{ACKNOWLEDGMENTS}

Fruitful discussions with Jorge Viñals are gratefully acknowledged. We wish to thank Thomas Erneux for useful comments. We acknowledge financial support from MCyT (Spain) and FEDER under Project Nos. BFM2001-0341C02-01 and BMF2000-1108.
[1] A. Nayfeh, Perturbation Methods (Wiley, New York, 1973).

[2] R. Graham, Phys. Rev. Lett. 76, 2185 (1996).

[3] A. Nayfeh, Introduction to Perturbation Techniques (Wiley, New York, 1981).

[4] A. Nayfeh, Nonlinear Oscillations (Wiley, New York, 1995).

[5] D. W. Hughes and M. R. E. Proctor, Physica D 46, 163 (1990).

[6] A. Daffertshofer, Phys. Rev. E 58, 327 (1998).

[7] M. Scalerandi, A. Romano, and C. A. Condat, Phys. Rev. E 58, 4166 (1998).

[8] G. De Vries and A. Sherman, J. Theor. Biol. 207, 513 (2000).

[9] G. E. Falkovich, I. Kolokolov, V. Lebedev, and S. K. Turitsyn, Phys. Rev. E 63, 025601 (2001).

[10] W. Genovese, M. A. Muñoz, and P. L. Garrido, Phys. Rev. E 58, 6828 (1998).

[11] P. Arnold, Phys. Rev. E 61, 6091 (2000).

[12] P. Arnold, Phys. Rev. E 61, 6099 (2000).

[13] E. Stone and P. Holmes, Physica D 37, 20 (1989).

[14] R. Kuske and G. Papanicolaou, Physica D 120, 255 (1998).

[15] R. Kuske, J. Stat. Phys. 96, 797 (1999).

[16] E. Stone and D. Armbruster, Chaos 9, 499 (1999).
[17] F. Drolet and J. Viñals, Phys. Rev. E 64, 026120 (2001).

[18] D. Bloömker, S. Maier-Paape, and G. Schneider, Discrete Contin. Dyn. Syst., Ser. B 1, 527 (2001).

[19] F. Drolet and J. Viñals, Phys. Rev. E 57, 5036 (1998).

[20] H. Risken, The Fokker-Planck Equation (Springer-Verlag, Berlin, 1989).

[21] M. San Miguel and R. Toral, in Stochastic Effects in Physical Systems, Instabilities and Nonequilibrium Structures Vol. VI, edited by E. Tirapegui and W. Zeller (Kluwer Academic Publisher, Dordrecht, 1997).

[22] J. Guckenheimer and P. Holmes, Nonlinear Oscillations, Dynamical Systems and Bifurcations of Vector Fields (SpringerVerlag, Berlin, 1983).

[23] R. Graham, in Weak Noise Limit and Nonequilibrium Potential of Dissipative Dynamical Systems, Instabilities and Nonequilibrium Structures Vol. I, edited by E. Tirapegui and W. Zeller (Reidel, Dordrecht, 1987).

[24] R. Graham, in Nonequilibrium Potentials in Spatially Extended Pattern Forming Systems, in Instabilities and Nonequilibrium Structures Vol. III, edited by E. Tirapegui and W. Zeller (Reidel, Dordrecht, 1991). 